\title{
AUTO-EFFICACITE CHEZ LES MERES ALLAITANTES DE NOUVEAU-NES PREMATURES
}

\author{
Rosely Aparecida Prandi Perrone \\ Clinical and Hospital Psychologist \\ PhD. student of Clinical Psychology, Faculty of Psychology, Lisbon University \\ roseprandi@hotmail.com
}

Recepción Artículo: 12 abril 2021

Admisión Evaluación: 12 abril 2021

Informe Evaluador 1: 14 abril 2021

Informe Evaluador 2: 18 abril 2021

Aprobación Publicación: 29 abril 2021

\section{RÉSUMÉ}

Le lait maternel est reconnu comme aliment idéal pour les nouveau-nés, y compris les prématurés, avec des avantages nutritionnels, biologiques, émotionnels et socio-économiques pour l'enfant et la famille. Pour les bébés prématurés, l'allaitement joue un rôle essentiel dans la mesure où il est associé à une réduction du temps d'hospitalisation et à divers états cliniques résultant d'une prématurité. Les études indiquent un faible taux d'allaitement parmi les mères de nouveau-nés prématurés et, souvent, un sevrage précoce. Actuellement, la littérature identifie l'auto-efficacité dans l'allaitement comme une variable importante qui influe sur les résultats de la réussite de l'allaitement. Afin d'identifier le principal instrument dédié à l'évaluation de l'auto-efficacité chez les mères allaitantes de bébés prématurés, une revue intégrative de la littérature a été réalisée sur les études, les évaluations et les instruments utilisés pour évaluer l'auto-efficacité chez les mères allaitantes des bébés prématurés. II a été constaté que la Breastfeeding Self-Efficacy Scale (BSES) est le principal instrument d'évaluation de la confiance d'une mère relativement à sa capacité d'allaiter. Pour ces mères, la Breastfeeding Self-Efficacy Scale-Short Form for Mothers of III or Preterm Infants (BSES-SFMIPI) permet de reconnaître les attentes et les expériences maternelles, d'identifier les mères de bébés prématurés à risque d'interrompre l'allaitement et aussi de proposer des stratégies individuelles pour encourager les mères et allaiter avec succès ces enfants.

Mots-clés: auto-efficacité; allaitement; nouveau-né prématuré; évaluation de l'allaitement

\section{ABSTRACT}

Self-efficacy in nursing mothers of premature infants. Breast milk is considered as the ideal food for newborns, including preterm babies, with nutritional, biological, emotional and socioeconomic benefits for the child and the family. For preterm babies, breastfeeding has an essential function because it is associated with the shortening of hospitalization time and various clinical conditions resulting from prematurity. Studies indicate a low breastfeeding rate among mothers of preterm babies and often early weaning. Currently, the literature identi- 


\section{AUTO-EFFICACITE CHEZ LES MERES ALLAITANTES DE NOUVEAU-NES PREMATURES}

fies breastfeeding self-efficacy as an important variable that affects outcomes in breastfeeding success. To identify the main instrument dedicated to the assessment of preterm mothers' breastfeeding self-efficacy, an integrative literature review was conducted about studies, evaluations and instruments for assessing self-efficacy in breastfeeding mothers of preterm babies. Breastfeeding Self-Efficacy Scale (BSES) was found to be the main instrument for assessing a mother's confidence in her ability to breastfeed. For these mothers, the Breastfeeding Self-Efficacy Scale-Short Form for Mothers of III or Preterm Infants (BSES-SFMIPI) was especially developed, enabling the recognition of maternal expectations and experiences, allowing the identification of mothers of preterm babies who are at risk of interrupting breastfeeding, and favoring the development of individual strategies to encourage and successfully breastfeed these babies.

Keywords: self-efficacy; breastfeeding; preterm babies; breastfeeding assessment

\section{INTRODUCTION}

Les évidences concernant l'importance de l'allaitement maternel exclusif sont concluants, non seulement pour la dyade mère-bébé mais, aussi pour tout le cycle de vie, y compris le large éventail de situations qui se développent depuis les premiers mois jusqu'au domaine des maladies chroniques à l'âge adulte, entraînant des bénéfices à court et à long terme (Caminha, Serva, Arruda, \& Batista Filho, 2010; Marques, \& Ramalho, 2015).

Le lait maternel est reconnu comme l'aliment idéal pour les nouveau-nés, y compris les prématurés, avec des avantages nutritionnels, biologiques, émotionnels et socio-économiques pour l'enfant et la famille. La World Health Organization (WHO) le recommande exclusivement au cours des six premiers mois de la vie et autant que possible jusqu'à l'âge de deux ans (WH0, 2001; 2018).

Les nouveau-nés prématurés (NNPT) constituent une population à haut risque et la prématurité est une cause majeure de morbidité et de mortalité, même dans les pays développés (Gharib, Fletcher, Tucker, Vohr, \& Lechner, 2018). Selon la WHO (2018), les taux de prématurité ont augmenté au cours des vingt dernières années et le pourcentage de survie des NNPT dépend du lieu de naissance. Chaque année, environ un million de bébés prématurés meurent dans le monde. Les bébés qui survivent peuvent avoir des incapacités permanentes, en particulier celles liées à l'apprentissage et aux problèmes visuels et auditifs, ce qui entraîne un coût élevé pour leurs familles et la société.

Outre les nombreux avantages offerts à tous les enfants, l'allaitement est associé à des revenus supplémentaires, en particulier pour les bébés nés prématurément. Pour ces nourrissons, l'allaitement joue un rôle essentiel, principalement parce qu'il est associé à une réduction du temps d'hospitalisation et à des conditions cliniques différentes dues à la prématurité (Pineda, 2010). Cependant, avec la séparation brutale de la mère à la naissance, le NNPT est privé de tout ce qu'un bébé né à terme reçoit en matière de soins maternels y compris l'allaitement (Ministério da Saúde do Brasil, 2017).

Malgré toutes les évidences scientifiques concernant les avantages de l'allaitement pour les NNPT, ces nourrissons font face à des défis importants en matière d'allaitement (Gharib et al., 2018).

Des études indiquent que la taux d'allaitement est faible chez les mères des prématurées et, souvent, le sevrage est précoce, non seulement à cause de l'état clinique du bébé mais, aussi, en raison des sentiments maternels de peur et d'insécurité (Castelli, 2017). En même temps, les mères de bébés prématurés, en particulier ceux dont le bébé est hospitalisé à l'unité néonatale de soins intensifs (UNSI), vivent un processus de souffrance avec des défis importants pour la santé mentale tels que le niveau élevé d'anxiété, de dépression et de stress (Camarneiro, \& Justo, 2014; Greene et al., 2015; Holditch-Davis et al., 2015; Kim et al., 2015) que, à son tour, nuisent à l'allaitement (Fallon, Groves, Halford, Bennett, \& Harrold, 2016; Wouk, Stuebe, \& Meltzer-Brody, 2017).

Une autre caractéristique de grande importance dans l'allaitement du bébé prématuré c'est le renforcement du lien affectif que fonctionne comme un stimulant pour la continuité de la production de lait maternelle dû au simple contact de la mère avec le bébé (Einloft, 2010). Cependant, des enquêtes à propos, d'une part, l'association des états d'anxiété et de dépression maternelle et, d'autre part, qualité de l'interaction avec NNPT ont montré des indicateurs négatifs qui compromettent la relation mère-bébé (Carvalho, Justo, \& Silva, 2018; Ryding, 2008). 
De plus, le manque de confiance de la mère en l'allaitement est un facteur de risque, en particulier, pour les mères de bébés NNPT dont les particularités de maturation rendent le processus de lactation difficile. Actuellement, la littérature identifie l'auto-efficacité dans l'allaitement comme une variable importante qui affecte les résultats sur le succès de l'allaitement (Wheeler, \& Dennis, 2013).

Des recherches scientifiques montrent que les mères avec un bas niveau de confiance en l'allaitement ont un risque plus élevé de l'interrompre tôt que les mères qui ont un niveau de confiance élevé (Awano, \& Shimada, 2010). De cette manière, l'auto-efficacité peut être une composante de la motivation liée à la performance et à la persistance, jouant un rôle important dans l'acquisition et le changement de comportement (Margotti, \& Epifanio, 2014).

La théorie de l'auto-efficacité a été utilisée dans plusieurs domaines et, plus particulièrement, dans les services de santé, pour la structuration de programmes. Ceux-ci peuvent servir de guides pour faciliter l'efficacité personnelle, que ce soit pour l'élaboration et l'utilisation d'échelles d'auto-efficacité identifiant les individus les plus à risque ou avec des difficultés à acquérir de nouvelles habitudes de santé. Ils peuvent également être utilisés pour la promotion des essais d'auto-efficacité de manière continue tout au long des programmes de promotion de la santé. De cette manière, il est possible de reconnaître les environnements et les moments les risqués de perte le sentiment d'efficacité personnelle qui amènent l'individu à abandonner ses efforts (Figueiredo, 2009).

La perception de auto-efficacité à propos de l'allaitement est une variable expressive, en particulier en ce qui concerne la durée de l'allaitement, car il est utile de prédire si la mère choisit d'allaiter ou non, combien d'efforts est-elle prête à dépenser, si elle va persister et comment elle répondra émotionnellement aux difficultés d'allaitement (Dennis, 1999).

La recherche sur l'allaitement prématuré et les nourrissons de faible poids à la naissance rapporte régulièrement que de nombreuses mères interrompent l'allaitement avant six mois (Wheeler, \& Dennis, 2013), une période recommandée par la WHO (2001) pour persévérer l'allaitement. Considérant que l'allaitement est essentiel pour les NNPT (Abrams, Schanler, Lee, \& Rechtman, 2014; Cristofalo et al., 2013; Sullivan et al., 2010) et que, en même temps, il est un défi pour les mères de ces bébés (Alves, Magano, Amorim, Nogueira, \& Silva, 2016; Hurst, Engebretson, \& Mahoney, 2013; Ikonen, Paavilainen, \& Kaunonen, 2015), connaître la motivation de la mère pour l'allaitement peut être un moyen d'identifier les difficultés ou les faiblesses de la mère, permettant ainsi la mise en place d'interventions pour soutenir le succès de l'allaitement (Souza, \& Fernandes, 2014).

\section{OBJECTIF}

Identifier le principal instrument dédié à l'évaluation de l'auto-efficacité chez les mères allaitantes de bébés NNPT.

\section{MÉTHODE}

\section{Conception}

La méthodologie de cette étude est constituée par une revue intégrative de la littérature qui permet la recherche, l'évaluation critique et la synthèse des preuves disponibles sur le sujet étudié, son produit final étant l'état actuel des connaissances sur le sujet étudié (Melnyk, 2003).

\section{Procédure}

Une recherche a été effectuée dans les principales bases de données d'études, d'évaluations et d'instruments, en utilisant les descripteurs suivants: "bébé prématuré", "allaitement", "auto-efficacité" et "évaluation". Les publications entre 1998 et 2018 en portugais, anglais et espagnol ont été inclus. Un autre critère d'éligibilité retenu a été l'utilisation d'une échelle ou d'un protocole d'évaluation de l'allaitement maternel des NNPTs.

Les informations recueillies ont été organisées en fonction de l'ordre chronologique de publication des instruments. Les informations suivantes ont été collectées: objectif, groupe d'âge spécifique évaluée, protocole utilisé, propriétés psychométriques et limitations relatives à l'application de l'instrument. 


\section{AUTO-EFFICACITE CHEZ LES MERES ALLAITANTES DE NOUVEAU-NES PREMATURES}

Les principales données recueillies dans les articles analysés concernaient le type d'instrument utilisé pour évaluer l'allaitement chez le NNPTs.

\section{RESULTATS}

Il a été constaté que la Breastfeeding Self-Efficacy Scale (BSES; Dennis, 1999) est l'outil le plus couramment utilisé pour évaluer la confiance de la mère à propos de sa capacité d'allaiter. Plus précisément, les études centrées sur l'allaitement maternel des NNPTs rapportent, toujours, que beaucoup de mères interrompent l'allaitement avant six mois.

Pour ces mères, la Breastfeeding Self-Efficacy Scale-Short Form for Mothers of III or Preterm Infants (BSESSFMIPI; Wheeler, \& Dennis, 2013) a été spécialement développé. II faut souligner que ces mères n'allaitent pas toujours directement au sein et que leurs bébés ont des comportements alimentaires et des besoins très différents de ceux des nouveau-nés en bonne santé nés à terme.

\section{DISCUSSION}

Cette recherche a révélé que l'allaitement au sein est particulièrement critique pour les NNPTs, une population qui fait face à des barrières physiologiques couplés avec les défis psychologiques de la mère pour le succès de l'allaitement (Gharib et al., 2018).

Dans de nombreuses enquêtes, les mères des NNPTs sont interrogées sur leurs pratiques d'allaitement au moment où leur bébé sort de l'hôpital. Le pourcentage de ces mères qui allaitent à la sortie varie entre $48 \%$ et $98 \%$. Ces mères sont plus susceptibles d'avoir des problèmes d'allaitement que les mères de bébés nés à terme. Les mères de NNPTs peuvent aussi éprouver un sentiment de culpabilité ou dépression liés aux obstacles à l'allaitement maternel, ce qui augmente leur niveau d'anxiété et de stress et diminue, encore plus, la production de lait (Akerstrom, Asplund, \& Norman, 2007; Callen, Pinelli, Atkinson, \& Saigal, 2005; Flacking, Nyqvist, Ewald, \& Wallin, 2003; Lee, Lee, \& Kuo, 2009; Tajanovska, Burns, \& Johnstone, 2007). Six mois après la sortie de l'hôpital, les taux d'allaitement chez les prématurés ont considérablement diminués (Flacking et al., 2003; Killersreiter, Grimmer, Buhrer, Dudenhausen, \& Obladen, 2001; Wheeler, \& Dennis, 2013).

D'autres problèmes d'allaitement chez les prématurés résultent de leur implication clinique, notamment de problèmes tels que des difficultés de respiration et de succion. (Callen et al., 2005). En outre, ces nourrissons sont généralement somnolents et peuvent manquer de la force et ou de l'endurance musculaire nécessaires pour sucer directement et efficacement sur le sein maternel (Callen et al., 2005; Reyna, Pickler, \& Thompson, 2006).

Considérant que l'auto-efficacité de l'allaitement est une variable que peux être changée, Wheeler et Dennis (2013) ont mis au point le BSES-SFMIPI, un instrument spécifique pour l'évaluation de l'auto-efficacité maternelle en matière d'allaitement au sein des NNPTs.

Pour évaluer l'échelle du point de vue psychométrique et pour examiner la relation entre l'auto-efficacité en matière d'allaitement et les variables sociodémographiques des mères et leurs bébés, Wheeler et Dennis (2013) ont développé une étude auprès de 144 mères de bébés malades et ou prématurés hospitalisés en UNSI.

La BSES-SFMIPI est une échelle d'auto-évaluation que vise à mesurer la confiance de la mère dans l'allaitement. La version originale a été validée par l'étude de Wheeler et Dennis (2013) auprès de la population des mères de nourrissons malades et ou prématurés. Cette échelle est composée de 18 items et divisée en deux domaines: domaine technique et pensée intrapersonnelle. Quatre items ont été ajoutés à la version originale de BSES-SF (Dennis, 2003) pour traiter les problèmes liés à l'extraction du lait maternel et à la transition à l'allaitement avec le sein maternel. Ces questions ont été posées pour aider à allaiter et pour déterminer quand le bébé devrait être nourri et aussi pour déterminer quand la mère doit changer d'un sein à l'autre. Les réponses doivent être enregistrées sur des échelles de Likertavec cinq alternatives variant de 1 (manque total de confiance) à 5 (confiance totale). Tous les éléments de l'échelle sont présentés de manière positive et les réponses sont résumées pour produire des résultats allant de 18 à 90. Les résultats les plus hauts indiquent des niveaux élevés d'auto-efficacité dans 
l'allaitement, suggérant ainsi une plus grande probabilité d'initier et de persévérer sur l'allaitement pendant une période plus longue.

Le coefficient alpha de Cronbach pour la cohérence interne de BSES-SFMIPI était de .88. La validité de l'échelle a été évaluée en comparant des groupes contrastés (mères qui continuent à allaiter et celles qui ont interrompu l'allaitement) et aussi en utilisant la corrélation entre ces groupes et les perceptions maternelles d'une production de lait insuffisante. L'appui à la validité prédictive a été démontré au moyen de différences significatives entre les moyennes chez les mères qui allaitaient ( $M=83.44, E T=8.23)$ et chez celles qui ont interrompu l'allaitement et l'extraction du lait maternel $(M=75.51, E T=10.08)$ six semaines après la sortie de l'hôpital.

Dans l'étude de Wheeler et Dennis (2013) visant à valider la BSES-SFMIPI, les résultats sociodémographiques maternels ont indiqué que l'âge moyen de l'échantillon de mères était de 29,8 ans (16-42), 95,8\% des femmes étaient mariées, 79,4\% étaient caucasiens, 14\% étaient autochtones, 53,1\% avaient un revenu économique élevé, 48,6\% ont eu un accouchement vaginal et 36,8\% avaient déjà eu une expérience d'allaitement.

Sur les 144 mères recrutées pour l'étude, $77,8 \%$ ont fourni des données sur l'allaitement jusqu'à six semaines après la sortie de l'hôpital du bébé. Les résultats pour les bébés indiquent un âge gestationnel moyen de 33,5 semaines (25-41) à la naissance et un poids moyen de $2.190 \mathrm{~g}(600 \mathrm{~g}-5103 \mathrm{~g})$ à la naissance. L'échantillon était composé à 80,7\% de NNPTs, à 6,1\% de bébés nés à terme et à 3,1\% de bébés nés après terme. Au total, 17 paires de jumeaux ont été trouvés (21\%). Les données ont également montré que, dans la population étudiée, $75,7 \%$ des nourrissons étaient sortis de I hôpital tandis qu'ils étaient nourris avec du lait maternel. Une semaine après sortir de l'hôpital, 90,8\% de ces bébés recevaient encore du lait maternel et 70,6\% ont été allaités jusqu'à la sixième semaine après leur sortie de l'hôpital. Ces taux d'allaitement sont similaires aux taux rapportés dans d'autres études sur des nouveau-nés malades ou prématurés (Wheeler, \& Dennis, 2013).

La recherche indique qu'il n'y avait pas d'association entre les variables démographiques maternelles et l'auto-efficacité dans l'allaitement, ce qui suggère que la BSES-SFMIPI est un outil efficace dans l'identification des mères de nourrissons malades ou prématurés avec un risque élevé d'interrompre tôt l'allaitement (Wheeler, \& Dennis, 2013).

L'analyse des résultats montre, aussi, qu'il n'y avait pas de différence dans les résultats d'auto-efficacité de l'allaitement au sein en fonction de l'origine ethnique, de l'âge de la mère et du type d'accouchement. Bien que il n'y avait pas de corrélation entre l'âge gestationnel du bébé à la naissance et l'auto-efficacité dans l'allaitement, une relation significative a été trouvée entre le poids à la naissance du bébé et les résultats sur la BSES-SFMIPI. Cette découverte est unique et cliniquement cohérente, témoignant que les bébés de faible poids à la naissance peuvent avoir plus de difficultés à allaiter et leurs mères peuvent avoir une auto-efficacité plus faible pour l'allaitement (Wheeler, \& Dennis, 2013).

Wheeler et Dennis (2013) ont constaté que le score moyen global sur la BSES-SFMIPI était de 79,39, le score maximal possible étant de 90 . Ce résultat est proportionnellement supérieur au score moyen $(55,8)$ sur la BSESSF originale où 70 est le maximum possible (Dennis, 2003). Cette comparaison suggère que les mères de nourrissons malades et ou prématurés une semaine après la sortie de l'hôpital étaient confiants dans leur capacité à allaiter, car beaucoup allaitaient depuis plusieurs semaines.

La conclusion la plus importante de l'étude de Wheeler et Dennis (2013) était liée à

la validité prédictive de BSES-SFMIPI. Les mères présentant des scores d'auto-efficacité d'allaitement élevés peu de temps après la sortie du bébé étaient plus susceptibles de poursuivre l'allaitement au bout de six semaines que les mères présentant des scores d'allaitement inférieurs à la sortie. Ce résultat est prometteur pour la pratique clinique, indiquant que les professionnels peuvent identifier les mères qui ont une faible auto-efficacité en matière d'allaitement et qui ont besoin d'un soutien supplémentaire avant leur sortie. II permet également la mise en place de soins individualisés pour répondre aux demandes de ces mères après la sortie du bébé, améliorant ainsi les résultats de la prévalence de l'allaitement. 


\section{AUTO-EFFICACITE CHEZ LES MERES ALLAITANTES DE NOUVEAU-NES PREMATURES}

Les résultats de l'étude de Wheeler et Dennis (2013) rendent compte de la validité de l'échelle confirment les rapports de la littérature qui identifient l'auto-efficacité en matière d'allaitement comme une variable importante qui interfère avec le succès de l'allaitement.

L'auto-efficacité fait référence à la perception que l'individu a sur ses capacités dans l'exercice d'une activité donnée (Barros, \& Batista-dos-Santos, 2010). Bandura (1994) comprend cette perception comme la croyance de l'individu en ses propres capacités à produire des niveaux de performance qui exercent une influence sur les événements de la vie. Ces croyances déterminent comment les gens se sentent, pensent, motivent et se comportent.

D'après le modèle d'auto-efficacité de Dennis en matière d'allaitement maternel (1999), il est confirmé que la confiance de la mère en sa capacité d'allaiter influence directement sa décision de commencer à allaiter et la durée de l'allaitement. En ce sens, l'application de la BSES-SFMIPI juste après l'accouchement de bébés NNPTs permit d'identifier, avec un degré de confiance élevé, les mères à risque de ne pas allaiter et ou d'interrompre tôt allaitement.

L'évaluation de l'auto-efficacité maternelle dans l'allaitement permit de savoir auparavant la thématique où la femme a le plus de difficulté, permettant, la mise en place de stratégies de soins et de promotion de l'allaitement maternel, avant que la mère décide de ne pas allaiter ou d'arrêter tôt l'allaitement.

Pour que l'allaitement maternel soit instauré et avec succès, les mères ont besoin d'un soutien actif pendant les périodes prénatales, d'accouchement et post-partum. L'un des défis les plus importants de la pratique de l'allaitement consiste à ménager aux divers facteurs qui influent sur la décision de la mère d'allaiter et de persévérer sur l'allaitement. Une mère bien informée et confiante est sans aucun doute le facteur principal du succès de l'allaitement au sein pour le bébé prématuré.

\section{CONCLUSION}

Les résultats ont montré que la BSES-SFMIPI est un outil particulièrement efficace pour identifier les mères de nouveau-nés malades et prématurés présentant un risque d'interruption prématurée de l'allaitement. De plus, la BSES-SFMIPI prouve qu'il s'agit d'une mesure valide et cohérente concernant la réalité psychique vécue par les mères de ces bébés.

\section{REFERENCES}

Abrams, S. A., Schanler, R. J., Lee, M. L., \& Rechtman, D. J. (2014). Greater mortality and morbidity in extremely preterm infants fed a diet containing cow milk protein products. Breastfeeding Medicine, 9(6), 281-285. doi:10.1089/bfm.2014.0024

Akerstrom, S., Asplund, I., \& Norman, M. (2007). Successful breastfeeding after discharge of preterm and sick newborn infants. Acta Paediatrica, 96, 1450-1454.

doi: 10.1111/j.1651-2227.2007.00502.x

Alves, E., Magano, R., Amorim, M., Nogueira, C., \& Silva, S. (2016). Factors influencing parent reports of facilitators and barriers to human milk supply in neonatal intensive care units. Journal of Human Lactation, 32(4), 695-703.

doi:10.1177/0890334416664071

Awano, M., \& Shimada K. (2010). Development and evaluation of a self-care program on breastfeeding in Japan: A quasi-experimental study. International Breastfeeding Journal. 5(9)1-10. http://www.internationalbreastfeedingjournal.com/content/5/1/9

Bandura, A. (1994). Self-efficacy. In: V. S. Ramachaudran (Ed.). Encyclopedia of human behavior (pp. 71-81). New York: Academic Press.

Barros, M., \& Batista-dos-Santos, A. C. (2010). Por dentro da autoeficácia: um estudo sobre seus fundamentos teóricos, suas fontes e conceitos correlatos. Revista Espaço Académico, 112, 1-9. http://periodicos.uem.br/ojs/index.php/EspacoAcademico/article/view/10818 
Callen, J., Pinelli, J., Atkinson, S., \& Saigal, S. (2005). Qualitative analysis of barriers to breastfeeding in verylow-birthweight infants in the hospital and post discharge. Advances in Neonatal Care, 5(2), 93-103.

https://www.medscape.com/viewarticle/502592

Camarneiro, A. P. F., \& Justo, J. M. R. M. (2014). 0 contributo dos fatores psicológicos na ocorrência do parto pré-termo. International Journal of Developmental and Educational Psychology, 1(4), 113-122. https://doi.org/10.17060/ijodaep.2014.n1.v4.595

Caminha, M. F. C., Serva, V. B., Arruda, I. K. G., \& Filho, M. B. (2010). Aspectos históricos, científicos, socioeconômicos e institucionais do aleitamento materno.

Revista Brasileira de Saúde Materno Infantil, 10(1), 25-37. http://dx.doi.org/10.1590/S1519-38292010000100003

Carvalho, M. E. S., Justo, J. M. R. M., \& Silva, H. M. F. R. (2018). Sound-Music Experiences and Maternal Sensitivity Following Preterm Birth: Contributions for the Model of Family-Centered Care. International Journal of Developmental and Educational Psychology, 1(2), 93-100.

https://doi.org/10.17060/ijodaep.2018.n2.v1.1336

Castelli, C. T. R. (2017). Avaliação da amamentação em recém-nascidos prematuros. Disseração de Mestrado, Universidade Federal de Ciências da Saúde de Porto Alegre, Porto Alegre, RGS, Brasil.

https://repositorio.ufcspa.edu.br/jspui/bitstream/123456789/499/1/Carla\%20Thamires\%20Rodriguez\%20Caste Ili_Dissertação.pdf

Cristofalo, E. A., Schanler, R. J., Blanco, C. L., Sullivan, S., Trawoeger, R., Kiechl-Kohlendorfer, U., . . A Abrams, S. (2013). Randomized trial of exclusive human milk versus preterm formula diets in extremely premature infants. Journal of Pediatrics, 163(6), 1592-1595. doi:10.1016/j.jpeds.2013.07.011

Dennis, C. L. (1999). Theoretical underpinnings of breastfeeding confidence: a self-efficacy framework. Journal of Human Lactation, 15, 195-201. https://doi.org/10.1177/089033449901500303

Einloft, P. R. (2010). Leite humano suplementado versus leite humano não suplementado na alimentação de recém-nascidos de muito baixo-peso: efeitos sobre a mineralização óssea e o crescimento. Tese de Doutorado, Pontifícia Universidade Católica do Rio Grande do Sul, Porto Alegre, RGS, Brasil.

http://repositorio.pucrs.br/dspace/handle/10923/4642

Fallon, V., Groves, R., Halford, J. C., Bennett, K. M., \& Harrold, J. A. (2016). Postpartum anxiety and infant-feeding outcomes: A systematic review. Journal of Human Lactation, 32(4), 740758. doi:10.1177/0890334416662241

Figueiredo, M. P. (2009). Contributos para a validação da escala de auto - eficácia na amamentação para português. Disseração de Mestrado, Instituto Superior de Psicologia Aplicada, Lisboa, Portugal. http://hdl.handle.net/10400.12/4627

Flacking, R., Nyqvist, K. H., Ewald, U., \& Wallin, L. (2003). Long-term duration of breastfeeding in Swedish low birth weight infants. Journal of Human Lactation, 19, 157-165. doi: 10.1177/0890334403252563

Greene, M. M., Rossman, B., Patra, K., Kratovil, A. L., Janes, J. E., \& Meier, P. P. (2015). Depression, anxiety, and perinatal specific posttraumatic distress in mothers of very low birth weight infants in the neonatal intensive care unit. Journal of Developmental and Behavioral Pediatrics, 36(5), 362-370. doi:10.1097/DBP.0000000000000174

Gharib, S., Fletcher, M., Tucker, R., Vohr, B., \& Lechner, B. E. (2018). Effect of Dedicated Lactation Support Services on Breastfeeding Outcomes in Extremely-Low-Birth-Weight Neonates. Journal of Human Lactation, 34(4) $728-736$.

doi: $0.1177 / 0890334417741304$ journals.sagepub.com/home/jhl

Holditch-Davis, D., Santos, H., Levy, J., White-Traut, R., O'Shea, T. M., Geraldo, V., \& David, R. (2015). Patterns of psychological distress in mothers of preterm infants. Infant Behavior and Development, 41, 154-163. doi: 10.1016/j.infbeh.2015.10.004 
Hurst, N., Engebretson, J., \& Mahoney, J. S. (2013). Providing mother's own milk in the context of the NICU: A paradoxical experience. Journal of Human Lactation, 29(3), 366-373. doi:10.1177/0890334413485640

Ikonen, R., Paavilainen, E., \& Kaunonen, M. (2015). Preterm infants' mothers' experiences with milk expression and breastfeeding: An integrative review. Advances in Neonatal Care, 15(6), 394-406. doi:10.1097/ANC.0000000000000232

Killersreiter, B., Grimmer, I., Buhrer, C., Dudenhausen, J., \& Obladen, M. (2001). Early cessation of breast milk feeding in very low birth weight infants. Early Human

Development, 60, 193-205. doi: 10.1016/S0378-3782(00)00116-X

Kim, W. J., Lee, E., Kim, K. R., Namkoong, K., Park, E. S., \& Rha, D. W. (2015). Progress of PTSD symptoms following birth: A prospective study in mothers of high-risk infants. Journal of Perinatology, 35(8), 575-579. doi:10.1038/jp.2015.9

Lee, T. Y., Lee, T. T., \& Kuo, S. C. (2009). The experiences of mothers in breastfeeding their very low birth weight infants. Journal of Advanced Nursing, 65, 2523-2531.

doi: 10.1111/j.1365-2648.2009.05116.x

Margotti, E., \& Epifanio, M. (2014). Aleitamento materno exclusivo e a Escala de Autoeficácia na Amamentação. Revista da Rede de Enfermagem do Nordeste, 15(5), 771-779. doi: 10.15253/2175-6783.2014000500006

Marques, S. M. N., \& Ramalho, S. I. H. S. M. A. (2015). Aleitamento materno: um estudo descritivo, no centro de saúde da região oeste. International Journal of Developmental and Educational Psychology, 1(1), 33-40.

https://doi.org/10.17060/ijodaep.2015.n1.v1.252

Melnyk, B. M. (2003). Finding and appraising systematic reviews of clinical interventions: critical skills for evidence-based practice. Journal of Pediatric Nursing, 29(2), 147-149. https://www.ncbi.nlm.nih.gov/pubmed/12723829

Ministério da Saúde do Brasil (2017). Atenção humanizada ao recém-nascido: Método Canguru: manual técnico.

http://bvsms.saude.gov.br/bvs/publicacoes/atencao_humanizada_metodo_canguru_manual_3ed.pdf

Papinczak, T., \& Turner C. (2000). An analysis of personal and social factors influencing initiation and duration of breastfeeding in a large Queensland maternity hospital. Breastfeed Review, 8(1), 25-33. https://www.ncbi.nlm.nih.gov/pubmed/10842578

Pineda R. (2010). Predictors of breastfeeding and breastmilk feeding among very low birth weight infants. Breastfeeding Medicine Journal, 6(5), 1-5. https://doi.org/10.1089/bfm.2010.0010

Reyna, B. A., Pickler, R. H., \& Thompson, A. (2006). A descriptive study of mothers' experiences feeding their preterm infants after discharge. Advances in Neonatal Care, 6, 333-340. doi: 10.1016/j.adnc.2006.08.007

Ryding, E. L. (2008). Identify and treat perinatal depression: for the sake of the baby! Acta Pædiatrica, 97(6), 697698. https://doi.org/10.1111/j.1651-2227.2008.00798.x

Souza, E. F. C., \& Fernandes, R. Á. Q. (2014). Autoeficácia na amamentação: um estudo de coorte. Acta Paulista de Enfermagem, 27(5), 465-470.

http://dx.doi.org/10.1590/1982-0194201400076

Sullivan, S., Schanler, R. J., Kim, J. H., Patel, A. L., Trawoger, R., Kiechl-Kohlendorfer, U., . . Lucas, A. (2010). An exclusively human milk-based diet is associated with a lower rate of necrotizing enterocolitis than a diet of human milk and bovine milk-based products. Journal of Pediatrics, 156(4), 562-567.

doi:10.1016/j.jpeds.2009.10.040

Tajanovska, M., Burns, S., \& Johnstone, L. (2007). A retrospective study of breastfeeding outcomes in an Australian neonatal intensive care unit. Journal of Neonatal Nursing, 13(4), 150-154. doi: https://doi.org/10.1016/j.jnn.2007.05.005

Wheeler, B. J., \& Dennis, C-L. (2013). Psychometric Testing of the Modified Breastfeeding Self-Efficacy Scale (Short Form) Among Mothers of III or Preterm Infants. JOGNN, 42, 70-80. https://doi.org/10.1111/j.15526909.2012.01431.x 
World Health Organization (2001). Report of the expert consultation on the optimal duration of exclusive breastfeeding: conclusions and recommendations.

http://www.who.int/nutrition/publications/infantfeeding/WHO_NHD_01.09/en/

World Health Organization (2018). Alimentación del lactante y del niño pequeño. http://www.who.int/es/newsroom/fact-sheets/detail/infant-and-young-child-feeding

Wouk, K., Stuebe, A. M., \& Meltzer-Brody, S. (2017). Postpartum mental health and breastfeeding practices: An analysis using the 2010-2011 Pregnancy Risk Assessment Monitoring System. Maternal and Child Health Journal, 21(3), 636-647. doi:10.1007/s10995-016-2150-6 
